\title{
Pfaffian orientation and enumeration of perfect matchings for some Cartesian products of graphs *
}

\author{
Feng-Gen Lin and Lian-Zhu Zhang ${ }^{\dagger}$ \\ School of Mathematical Sciences, Xiamen University \\ Xiamen 361005, P.R.China \\ E-mail: zhanglz@xmu.edu.cn
}

Submitted: Dec 13, 2008; Accepted: Apr 14, 2009; Published: Apr 30, 2009

Mathematics Subject Classification(2000): 05A15; 05C70.

\begin{abstract}
The importance of Pfaffian orientations stems from the fact that if a graph $G$ is Pfaffian, then the number of perfect matchings of $G$ (as well as other related problems) can be computed in polynomial time. Although there are many equivalent conditions for the existence of a Pfaffian orientation of a graph, this property is not well-characterized. The problem is that no polynomial algorithm is known for checking whether or not a given orientation of a graph is Pfaffian. Similarly, we do not know whether this property of an undirected graph that it has a Pfaffian orientation is in NP. It is well known that the enumeration problem of perfect matchings for general graphs is NP-hard. L. Lovász pointed out that it makes sense not only to seek good upper and lower bounds of the number of perfect matchings for general graphs, but also to seek special classes for which the problem can be solved exactly. For a simple graph $G$ and a cycle $C_{n}$ with $n$ vertices (or a path $P_{n}$ with $n$ vertices), we define $C_{n}$ (or $\left.P_{n}\right) \times G$ as the Cartesian product of graphs $C_{n}$ (or $P_{n}$ ) and $G$. In the present paper, we construct Pfaffian orientations of graphs $C_{4} \times G, P_{4} \times G$ and $P_{3} \times G$, where $G$ is a non bipartite graph with a unique cycle, and obtain the explicit formulas in terms of eigenvalues of the skew adjacency matrix of $\vec{G}$ to enumerate their perfect matchings by Pfaffian approach, where $\vec{G}$ is an arbitrary orientation of $G$.
\end{abstract}

\section{Introduction}

The theory of Pfaffian orientations of graphs had been introduced by the physicists M. E. Fisher, P. W. Kasteleyn, and H. N. V. Temperley. The importance of Pfaffian orientations stems

*This work is supposed by NFSC (NO.10831001).

${ }^{\dagger}$ Corresponding author. 
from the fact that if a graph $G$ is Pfaffian, then the number of perfect matchings of $G$ (as well as other related problems) can be computed in polynomial time.

P. W. Kasteleyn gave a polynomial time algorithm for computing the number of perfect matchings in planar graphs using Pfaffian method and extended his approach to toroidal grids in [6] and [8]. Litte [9] generalized P. W. Kasteleyn's work and proved that if a bipartite graph $G$ contains no subdivision of $K_{3,3}$, then $G$ has a Pfaffian orientation. Furthermore, Fischer and Little [3] proved that a graph has a Pfaffian orientation under which every cycle of even length is clockwise odd if and only if the graph contains no subgraph which is, after the contraction of at most one cycle of odd length, an even subdivision of $K_{2,3}$. McCuaig [11], and McCuaig, Robertson et al [12], and Robertson, Seymour et al [15] found a polynomial-time algorithm to determine whether a bipartite graph has a Pfaffian orientation respectively. In spite of there are many equivalent conditions for the existence of a Pfaffian orientation of a graph, this property is not well-characterized. The problem is that no polynomial algorithm is known for checking whether or not a given orientation of a graph $G$ is Pfaffian. We do not even know whether this property is in NP. (It is trivially in co-NP; to prove that a given orientation is non-Pfaffian, it suffices to exhibit two perfect matchings with different signs.) similarly, we do not know whether the property of an undirected graph that it has a Pfaffian orientation is in NP.

The number of perfect matchings is an important topological index which has been applied for estimation of the resonant energy and total $\pi$-electron energy and calculation of pauling bond order (see [4], [13], [16]). Enumeration problem for perfect matchings in general graphs(even in bipartite graph) is NP-hard. L. Lovász [10] pointed out that it makes sense not only to seek good upper and lower bounds of the number of perfect matchings for general graphs, but also to seek special classes for which the problem can be solved exactly. So far, many mathematicians, physicists and chemists have focused most of their attention on the enumeration problem for perfect matchings (see [2], [3], [5], [14], [15]).

First, we repeat some standard definitions. A graph $G$ is a pair $V(G)$ and $E(G)$, where $V(G)$ is a finite set of vertices and $E(G)$ is a set of unordered pairs $x y$ of vertices called edges. We say that the edge $x y$ is incident with $x, y$ and that $x, y$ are adjacent and are called the ends of the edge $x y$. All graphs in this paper are simple graphs which are finite, do not have loops or multiple edges. A graph $H$ is a subgraph of $G$ if $V(H) \subseteq V(G)$ and $E(H) \subseteq E(G)$. A spanning subgraph of $G$ is a subgraph $H$ with $V(H)=V(G)$. For a nonempty subset $V^{\prime}$ of $V(G)$, a subgraph of $G$ is called induced subgraph induced by $V^{\prime}$ if its vertex set is $V^{\prime}$ and edge set is the set of those edges of $G$ that have both ends in $V^{\prime}$. A $k$-path denoted by $x_{0} x_{1} \ldots x_{k}$ is the graph with distinct vertices $x_{0}, x_{1}, \ldots, x_{k}$ and edges $x_{i-1} x_{i}, i=1,2, \ldots, k$, where $x_{0}, x_{k}$ are called its ends. A $k$-cycle is obtained from a $(k-1)$-path by adding the edge between the two ends $x_{0}, x_{k-1}$. We say that $k$ is the length of the $k$-path and $k$-cycle. A path and a cycle with $n$ vertices are denoted by $P_{n}$ and $C_{n}$ respectively. A graph is connected if any two vertices are joined by a path. A bipartite graph is one whose vertex set can be partitioned into two disjoint subsets $X$ and $Y$, so that each edge has one end in $X$ and one end in $Y$; such a partition $(X, Y)$ is called a bipartition of the graph. A graph is bipartite if and only if each cycle of it has even length. A tree is a connected acyclic graph. Clearly, a tree is bipartite. A perfect matching of a simple graph $G$ is a set of vertex-disjoint edges that are collectively incident to all vertices. A cycle $C$ of $G$ is said to be 
nice if $G-C$ contains a perfect matching, where $G-C$ denotes the induced subgraph of $G$ obtained from $G$ by deleting the vertices of $C$. If $C$ is a nice cycle of a spanning subgraph of $G$, then $C$ is also a nice cycle of $G$. Let $\vec{G}$ denote an orientation of $G$ which is obtained from $G$ by specifying, for each edge, an orientation on its ends. If $C$ is an even undirected cycle in $\vec{G}$, we shall say $C$ is evenly oriented if it has an even number of edges oriented in the direction of the routing. Otherwise $C$ is oddly oriented. An orientation $\vec{G}$ is Pfaffian if every nice cycle of $G$ is oddly oriented in $\vec{G}$. A graph $G$ is Pfaffian if it has a Pfaffian orientation.

For $V(G)=\left\{v_{1}, v_{2}, \ldots, v_{n}\right\}$, the skew adjacency matrix of $\vec{G}$, denoted by $A(\vec{G})$, is defined as follows:

$$
A(\vec{G})=\left(a_{i j}\right)_{n \times n}, \quad \text { where } \quad a_{i j}= \begin{cases}1 & \text { if }\left(v_{i}, v_{j}\right) \in E(\vec{G}), \\ -1 & \text { if }\left(v_{j}, v_{i}\right) \in E(\vec{G}), \\ 0 & \text { otherwise. }\end{cases}
$$

Let $\overleftarrow{G}$ be the reverse orientation of $\vec{G}$ such that $A(\overleftarrow{G})=-A(\vec{G})$

The cartesian product of two graphs $G$ and $H$ denoted by $G \times H$ is the graph with vertex set $V(G) \times V(H)$ such that $(x, u)$ and $(y, v)$ are adjacent iff either $x=y$ and $u$ and $v$ are adjacent in $H$, or $u=v$ and $x$ and $y$ are adjacent in $G$. For bipartite graphs, Yan and Zhang in [18] studied the enumeration of perfect matchings for these Cartesian product of graphs $C_{4} \times T, P_{4} \times T$ and $P_{3} \times T$, where $T$ is a tree. In the present paper, we construct Pfaffian orientations of some Cartesian product of graphs which are non-bipartite and obtain explicit formulas to enumerate their perfect matchings by Pfaffian approach as follows.

(1) $\phi\left(C_{4} \times G\right)=\prod_{\lambda \in \lambda(\vec{G})}\left(2-\lambda^{2}\right) ;(2) \phi\left(P_{4} \times G\right)=\prod_{\lambda \in \lambda^{*}(\vec{G})}\left(1-3 \lambda^{2}+\lambda^{4}\right)$;

(3) If $G$ has a perfect matching, then $\phi\left(P_{3} \times G\right)=\prod_{\lambda \in \lambda^{*}(\vec{G})}\left(2-\lambda^{2}\right)$ and $\phi\left(C_{4} \times G\right)=\phi\left(P_{3} \times G\right)^{2}$,

where $G$ is a non-bipartite graph with a unique cycle, $\vec{G}$ is an arbitrary orientation of $G, \lambda(\vec{G})$ is the set of all eigenvalues of $A(\vec{G})$ and $\lambda^{*}(\vec{G})$ is the set of those non-negative imaginary part eigenvalues of $A(\vec{G})$.

\section{Pfaffian orientation}

Theorem 2.1 [10]. Let $G$ be any simple graph with even number of vertices, and $\vec{G}$ be an orientation of $G$. Then the following three properties are equivalent:

(1) $\vec{G}$ is a Pfaffian orientation.

(2) Every nice cycle in $G$ is oddly oriented relative to $\vec{G}$.

(3) If $G$ has a perfect matching, then for some perfect matching $F$, every $F$-alternating cycle is oddly oriented relative to $\vec{G}$.

For a simple graph $G$ with $V(G)=\left\{v_{1}, v_{2}, \ldots, v_{n}\right\}$, let $G_{1}$ and $G_{2}$ be two copies of $G$ with $V\left(G_{1}\right)=\left\{v_{1}^{\prime}, v_{2}^{\prime}, \ldots, v_{n}^{\prime}\right\}$ and $V\left(G_{2}\right)=\left\{v_{1}^{\prime \prime}, v_{2}^{\prime \prime}, \ldots, v_{n}^{\prime \prime}\right\}$ respectively, where $v_{i}^{\prime}$ in $G_{1}$ and $v_{i}^{\prime \prime}$ 
in $G_{2}$ are corresponding to $v_{i}$ in $G(i=1,2, \cdots, n)$. Adding the edges $v_{i}^{\prime} v_{i}^{\prime \prime}(i=1,2, \cdots, n)$ between $G_{1}$ and $G_{2}$, the resulting graph is $P_{2} \times G$ with vertex set $V\left(G_{1}\right) \cup V\left(G_{2}\right)$ and edge set $E\left(G_{1}\right) \cup E\left(G_{2}\right) \cup\left\{v_{i}^{\prime} v_{i}^{\prime \prime} \mid i=1,2, \cdots, n\right\}$. If $\vec{G}$ is an orientation of $G$, then we denote the orientation of $P_{2} \times G$ by $\left(P_{2} \times \vec{G}\right)^{e}$ which is defined as follows: the orientation of $G_{1}$ (the left half of $\left.P_{2} \times G\right)$ is the same as $\vec{G}$ and that of $G_{2}$ (the right half of $P_{2} \times G$ ) is the same as $\overleftarrow{G}$, and the orientations of edges $v_{i}^{\prime} v_{i}^{\prime \prime}(i=1,2, \cdots, n)$ are from $v_{i}^{\prime}$ to $v_{i}^{\prime \prime}$ (see Figure 1$)$.

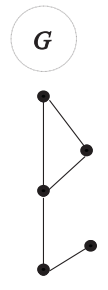

(a) $G$

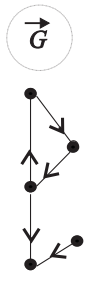

(b) $\vec{G}$

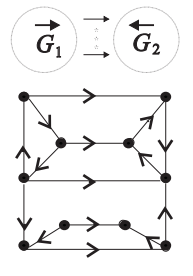

(c) $P_{2} \times \vec{G}$

Figure 1.

Lemma 2.2 [17]. Let $G$ be a simple graph. If $\vec{G}$ is an orientation of $G$ under which every cycle of even length is oddly oriented in $\vec{G}$, then the orientation $\left(P_{2} \times \vec{G}\right)^{e}$ is a Pfaffian orientation of $P_{2} \times G$.

Lemma 2.3 [18]. Suppose $G$ is a tree with $V(G)=\left\{v_{1}, v_{2}, \ldots, v_{n}\right\}$. Then every cycle $C$ of $P_{2} \times G$ is a nice cycle and can be written as:

$$
v_{i_{1}}^{\prime} v_{i_{2}}^{\prime} \ldots v_{i_{m}}^{\prime} v_{i_{m}}^{\prime \prime} v_{i_{m-1}}^{\prime \prime} \ldots v_{i_{2}}^{\prime \prime} v_{i_{1}}^{\prime \prime} v_{i_{1}}^{\prime}
$$

where $i_{1}, i_{2}, \ldots, i_{m} \in\{1,2, \ldots, n\}$.

Lemma 2.4. If $G$ is a tree with $V(G)=\left\{v_{1}, v_{2}, \ldots, v_{n}\right\}$, then any two paths $P_{v_{i}^{\prime}-v_{j}^{\prime \prime}}$ and $P_{v_{j}^{\prime}-v_{i}^{\prime \prime}}$ for $i \neq j$ must intersect each other in $P_{2} \times G, i, j \in\{1,2, \ldots, n\}$.

Proof. We prove this assertion by contradiction. If there exist two disjoint paths $P_{v_{i}^{\prime}-v_{j}^{\prime \prime}}$ and $P_{v_{j}^{\prime}-v_{i}^{\prime \prime}}$ for $i \neq j$ in $P_{2} \times G$, then there is a cycle of $P_{2} \times G$ that consists of $P_{v_{i}^{\prime}-v_{j}^{\prime \prime}}, v_{j}^{\prime \prime} v_{j}^{\prime}, P_{v_{j}^{\prime}-v_{i}^{\prime \prime}}$ and $v_{i}^{\prime \prime} v_{i}^{\prime}$, which has the following form:

$$
v_{i}^{\prime} \ldots \ldots v_{j}^{\prime \prime} v_{j}^{\prime} \ldots \ldots v_{i}^{\prime \prime} v_{i}^{\prime}
$$

In the other hand, by Lemma 2.3, every cycle of $P_{2} \times G$ has the form (1) in Lemma 2.3. It is clear that the cycle form (2) is distinct from the cycle form (1), a contradiction. The assertion holds.

In order to formulate our main results, it is necessary to introduce further terminology.

Suppose $G$ is a non-bipartite graph with a unique cycle. For convenience, let $C^{*}$ denote the unique odd cycle of $G$ with length $2 k+1$, and label the vertices of $G$ as $v_{1}, v_{2}, \ldots, v_{2 k+1}, \ldots, v_{n}$ such that $C^{*}=v_{1} v_{2} \ldots v_{2 k+1} v_{1}$ (see Figure 2(a)). In $P_{2} \times G\left(\right.$ see Figure $2(\mathrm{~b})$ ), let the cycles $C_{i}^{*}$ in $G_{i}(\mathrm{i}=1,2)$ be corresponding to the cycle $C^{*}$ in $G$. For $E^{\prime} \subseteq E(G), G-E^{\prime}$ denotes the graph obtained from $G$ by deleting the edges in $E^{\prime}$. If $E^{\prime}=\{e\}$ we write $G-e$ instead of $G-\{e\}$. A path with ends $s$ and $t$ is denoted by $P_{s-t}$. 


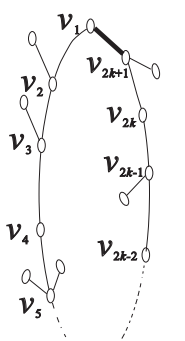

(a) $G$

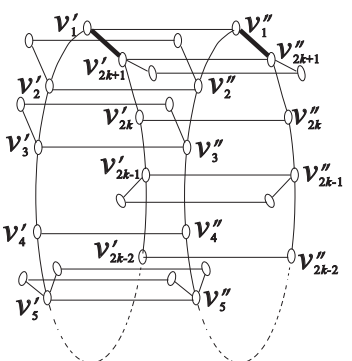

(b) $P_{2} \times G$

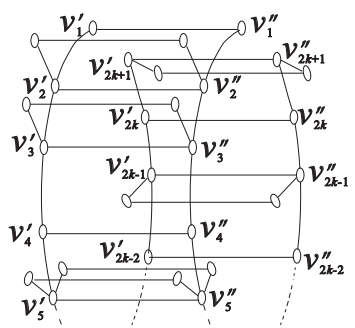

(c) $P_{2} \times\left(G-v_{1} v_{2 k+1}\right)$

Figure 2.

Theorem 2.5. If $G$ is a non-bipartite graph with a unique cycle, then every even cycle of $P_{2} \times G$ is a nice cycle of it.

Proof. We only need to prove the case that $G$ is a connected graph. Suppose $\widetilde{C}$ is an even cycle in $P_{2} \times G$, and $E^{\prime}=\left\{v_{1}^{\prime} v_{2 k+1}^{\prime}, v_{1}^{\prime \prime} v_{2 k+1}^{\prime \prime}\right\}$. If $\widetilde{C}$ contains no edge of $E^{\prime}$, then $\widetilde{C}$ is an even cycle of $P_{2} \times\left(G-v_{1} v_{2 k+1}\right)$ (see Figure 2(c)) which is a spanning subgraph of $P_{2} \times G$. Since $G-v_{1} v_{2 k+1}$ is a tree, $\widetilde{C}$ is a nice cycle of $P_{2} \times\left(G-v_{1} v_{2 k+1}\right)$ by Lemma 2.3. Thus $\widetilde{C}$ is a nice cycle of $P_{2} \times G$.

If $\widetilde{C}$ contains exactly one edge of $E^{\prime}$, without loss of generality, we assume that $\widetilde{C}$ contains the edge $v_{1}^{\prime} v_{2 k+1}^{\prime}$. Since $G-v_{1} v_{2 k+1}$ is a tree, it is bipartite. If $\left(V_{1}, V_{2}\right)$ is its a bipartition, then $P_{2} \times\left(G-v_{1} v_{2 k+1}\right)$ is a bipartite graph with a bipartition $\left(V_{1}^{\prime} \cup V_{2}^{\prime \prime}, V_{2}^{\prime} \cup V_{1}^{\prime \prime}\right)$, where both $V_{i}^{\prime}$ and $V_{i}^{\prime \prime}$ are corresponding to $V_{i}, i=1,2$. Furthermore, there exists a $2 k$-path $v_{1}^{\prime} v_{2}^{\prime} \ldots v_{i}^{\prime} v_{i+1}^{\prime} \ldots v_{2 k}^{\prime} v_{2 k+1}^{\prime}$, so the two vertices $v_{1}^{\prime}, v_{2 k+1}^{\prime}$ belong to the same partitioned subset. Hence $\widetilde{C}-v_{1}^{\prime} v_{2 k+1}^{\prime}$ is a path $P_{v_{1}^{\prime}-v_{2 k+1}^{\prime}}$ of $P_{2} \times\left(G-v_{1} v_{2 k+1}\right)$ which always has even length. Thus the length of $\widetilde{C}$ is odd, a contradiction.

If $\widetilde{C}$ contains both edges of $E^{\prime}$, then $\widetilde{C}-E^{\prime}$ consists of two disjoint paths $P_{v_{1}^{\prime}-v_{1}^{\prime \prime}}$ and $P_{v_{2 k+1}^{\prime}-v_{2 k+1}^{\prime \prime}}$ of $P_{2} \times\left(G-v_{1} v_{2 k+1}\right)$. Otherwise, there are two disjoint paths $P_{v_{1}^{\prime}-v_{2 k+1}^{\prime \prime}}$ and $P_{v_{2 k+1}^{\prime}-v_{1}^{\prime \prime}}$ which contradicts to Lemma 2.4. By application of Lemma 2.3, we have $P_{v_{1}^{\prime}-v_{1}^{\prime \prime}}^{21}=v_{1}^{\prime} v_{1}^{\prime \prime}$ or $v_{1}^{\prime} v_{i_{1}}^{\prime} \ldots v_{i_{s}}^{\prime} v_{i_{s}}^{\prime \prime} \ldots v_{i_{1}}^{\prime \prime} v_{1}^{\prime \prime}$, and $P_{v_{2 k+1}^{\prime}-v_{2 k+1}^{\prime \prime}}=v_{2 k+1}^{\prime} v_{2 K+1}^{\prime \prime}$ or $v_{2 k+1}^{\prime} v_{j_{1}}^{\prime} \ldots v_{j_{t}}^{\prime} v_{i_{t}}^{\prime \prime} \ldots v_{j_{1}}^{\prime \prime} v_{2 k+1}^{\prime \prime}$ respectively. Thus, $\left\{v_{l}^{\prime} v_{l}^{\prime \prime} \mid 1 \leqslant l \leqslant n, v_{l}^{\prime} \notin V(\widetilde{C})\right\}$ is a perfect matching of $G-\widetilde{C}$. Therefore $\widetilde{C}$ is a nice cycle. $\square$

By Theorem 2.5, the following corollary is immediate.

Corollary 2.6. Every even cycle of $P_{2} \times C_{2 k+1}$ is a nice cycle of it.

Suppose $G$ is a non-bipartite graph with a unique cycle and $\vec{G}$ is an arbitrary orientation of $G$. By Lemma 2.2, the orientation $\left(P_{2} \times \vec{G}\right)^{e}$ is a Pfaffian orientation of $P_{2} \times G$. Hence every nice cycle in $\left(P_{2} \times \vec{G}\right)^{e}$ is oddly oriented by Theorem 2.1 . By Theorem 2.5 , the orientation $\left(P_{2} \times \vec{G}\right)^{e}$ is an orientation of $P_{2} \times G$ under which every even cycle of $P_{2} \times G$ is oddly oriented. Now we apply Lemma 2.2 with $G$ replaced by $P_{2} \times G$, then $\left(P_{2} \times\left(P_{2} \times \vec{G}\right)^{e}\right)^{e}$ is a Pfaffian orientation of $P_{2} \times\left(P_{2} \times G\right)$. Since $P_{2} \times P_{2}=C_{4}$, we use $\left(C_{4} \times \vec{G}\right)^{e}$ instead of $\left(P_{2} \times\left(P_{2} \times \vec{G}\right)^{e}\right)^{e}$ for convenience. Figure 3 illustrates the orientation procedure. 


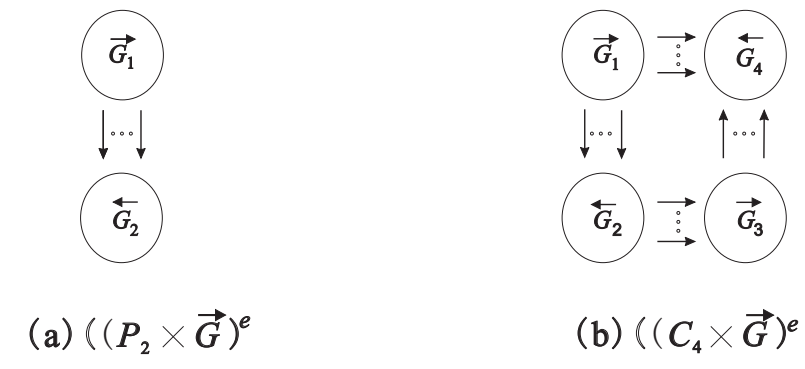

Figure 3.

For $G$ with $V(G)=\left\{v_{1}, v_{2}, \ldots, v_{n}\right\}$, take $m$ copies of $G$, denoted by $G_{i}$ with $V\left(G_{i}\right)=$ $\left\{v_{1}^{(i)}, v_{2}^{(i)}, \ldots, v_{n}^{(i)}\right\}, i=1,2, \ldots, m . P_{m} \times G$ is the graph with vertex set $\bigcup_{j=1}^{m} V\left(G_{i}\right)$ and edge set $\bigcup_{j=1}^{m} E\left(G_{i}\right) \cup\left\{v_{j}^{(i)} v_{j}^{(i+1)} \mid 1 \leqslant j \leqslant n, 1 \leqslant i \leqslant m-1\right\}$. Let $\vec{G}$ be an orientation of $G$. We define the orientation of $G_{i}$ in $P_{m} \times G$ to be the same as $\vec{G}$ if $i$ is odd, $\overleftarrow{G}$ otherwise, and the orientations of edges $v_{j}^{(i)} v_{j}^{(i+1)}$ in $P_{m} \times G$ to be from $v_{j}^{(i)}$ to $v_{j}^{(i+1)}(1 \leqslant j \leqslant n, 1 \leqslant i \leqslant m-1)$. The orientation of $P_{m} \times G$ defined as above is denoted by $\left(P_{m} \times \vec{G}\right)^{e}$. The processes of the orientations $\left(P_{3} \times \vec{G}\right)^{e}$ and $\left(P_{4} \times \vec{G}\right)^{e}$ are shown in Figure 4 .

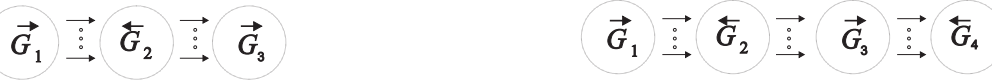

$$
\begin{aligned}
& \text { (a) }\left(P_{3} \times \vec{G}\right)^{e} \quad \text { (b) }\left(P_{4} \times \vec{G}\right)^{e}
\end{aligned}
$$

Figure 4.

Since $P_{4} \times G$ is a spanning subgraph of $C_{4} \times G$, every nice cycle in $P_{4} \times G$ is also a nice cycle in $C_{4} \times G$. Noting that $\left(P_{4} \times \vec{G}\right)^{e}$ is the orientation $\left(C_{4} \times \vec{G}\right)^{e}$ restricted in $P_{4} \times G$ and $\left(C_{4} \times \vec{G}\right)^{e}$ is a Pfaffian orientation, we obtain that every nice cycle in $P_{4} \times G$ is oddly oriented relative to $\left(P_{4} \times \vec{G}\right)^{e}$. Then we get the following theorem immediately.

Theorem 2.7. Let $G$ be a non-bipartite graph with a unique cycle, and $\vec{G}$ be an arbitrary orientation of $G$. Then the orientations $\left(C_{4} \times \vec{G}\right)^{e}$ of $C_{4} \times G$ and $\left(P_{4} \times \vec{G}\right)^{e}$ of $P_{4} \times G$ are Pfaffian orientations.

Theorem 2.8. Let $G$ be a non-bipartite graph with a unique cycle, and $\vec{G}$ be an arbitrary orientation of $G$. If $G$ has a perfect matching, then $\left(P_{3} \times \vec{G}\right)^{e}$ is a Pfaffian orientation of $P_{3} \times G$. Proof. Since $G$ has a perfect matching, it is clear that $P_{3} \times G$ has a perfect matching. Suppose $C$ is an arbitrary nice cycle of $P_{3} \times G$ and $M_{1}$ is a perfect matching of $P_{3} \times G-C$. Let $M_{2}$ be a perfect matching of $G_{4}$ in $P_{4} \times G$. Clearly $M_{1} \cup M_{2}$ is a perfect matching of $P_{4} \times G-C$. So that every nice cycle in $P_{3} \times G$ is also a nice cycle in $P_{4} \times G$. Moreover, $\left(P_{3} \times \vec{G}\right)^{e}$ is the orientation $\left(P_{4} \times \vec{G}\right)^{e}$ restricted in $P_{3} \times G$, and $\left(P_{4} \times \vec{G}\right)^{e}$ is a Pfaffian orientation by Theorem 2.7 , so every nice cycle in $P_{3} \times G$ is oddly oriented relative to $\left(P_{3} \times \vec{G}\right)^{e}$. By Theorem $2.1,\left(P_{3} \times \vec{G}\right)^{e}$ is a Pfaffian orientation. 


\section{Enumeration of perfect matchings}

If a graph $G$ has a Pfaffian orientation $\vec{G}$, then the number of perfect matchings of $G$ denoted by $\phi(G)$ can be computed in polynomial time by the following theorem.

Theorem 3.1 [7], [8], [10]. Let $\vec{G}$ be a Pfaffian orientation of a graph $G$. Then

$$
\phi(G)^{2}=\operatorname{det} A(\vec{G})
$$

where $A(\vec{G})$ is the skew adjacency matrix of $\vec{G}$.

Theorem 3.2. Let $G$ be a non-bipartite graph with a unique cycle, and $\vec{G}$ be an arbitrary orientation of $G$. Then

$$
\phi\left(C_{4} \times G\right)=\prod_{\lambda \in \lambda(\vec{G})}\left(2-\lambda^{2}\right),
$$

where $\lambda(\vec{G})$ is the set of all eigenvalues of $A(\vec{G})$.

Proof. $\left(C_{4} \times \vec{G}\right)^{e}$ is a Pfaffian orientation of $C_{4} \times G$ by Theorem 2.7. The skew adjacency matrix of $\left(C_{4} \times \vec{G}\right)^{e}$ has the following form by a suitable labeling of vertices of $\left(C_{4} \times \vec{G}\right)^{e}$ :

$$
A\left(\left(C_{4} \times \vec{G}\right)^{e}\right)=\left(\begin{array}{cccc}
A(\vec{G}) & I & I & 0 \\
-I & -A(\vec{G}) & 0 & I \\
-I & 0 & -A(\vec{G}) & -I \\
0 & -I & I & A(\vec{G})
\end{array}\right)=\left(\begin{array}{cc}
A & B \\
C & D
\end{array}\right)
$$

where $I$ is the identity matrix, $A=\left(\begin{array}{cc}A \overrightarrow{(G}) & I \\ -I & -A(\vec{G})\end{array}\right), B=\left(\begin{array}{cc}I & 0 \\ 0 & I\end{array}\right), C=\left(\begin{array}{cc}-I & 0 \\ 0 & -I\end{array}\right), D=$ $\left(\begin{array}{cc}-A(\vec{G}) & -I \\ I & A(\vec{G})\end{array}\right)$

It is well known that for four matrixes $A, B, C, D$ with equivalent order $n$, if $\operatorname{det} A \neq 0$ and $A C=C A$, then $\operatorname{det}\left(\begin{array}{cc}A & B \\ C & D\end{array}\right)=\operatorname{det}(A D-C B)$. By Theorem 3.1, we have

$$
\begin{aligned}
\phi\left(C_{4} \times G\right)^{2} & =\operatorname{det} A\left(\left(C_{4} \times \vec{G}\right)^{e}\right) \\
& =\operatorname{det}\left\{-\left(\begin{array}{cc}
A(\vec{G}) & I \\
-I & -A(\vec{G})
\end{array}\right)^{2}+\left(\begin{array}{cc}
I & 0 \\
0 & I
\end{array}\right)\right\} \\
& =\operatorname{det}\left(\begin{array}{cc}
2 I-(A(\vec{G}))^{2} & 0 \\
0 & 2 I-(A(\vec{G}))^{2}
\end{array}\right) \\
& =\left(\operatorname{det}\left(2 I-A(\vec{G})^{2}\right)\right)^{2} .
\end{aligned}
$$

Since $A(\vec{G})$ is a real skew matrix, its eigenvalues are either zeros or pure imaginary numbers, 
hence

$$
\phi\left(C_{4} \times G\right)=\left|\operatorname{det}\left(2 I-A(\vec{G})^{2}\right)\right|=\prod_{\lambda \in \lambda(\vec{G})}\left(2-\lambda^{2}\right)
$$

where $\lambda(\vec{G})$ is the set of all eigenvalues of $A(\vec{G})$.

Corollary 3.3. Let $G$ be an odd cycle with $2 k+1$ vertices. Then

$$
\phi\left(C_{4} \times G\right)=\prod_{j=1}^{2 k+1}\left(2+4 \sin ^{2}\left(\frac{2 j \pi}{2 k+1}\right)\right) .
$$

Proof. Without loss of generality, we orient every edge of the odd cycle $G$ clockwise. Then the skew adjacency matrix $A(\vec{G})$ is a circulant matrix [1], and the eigenvalues of $A(\vec{G})$ are $\lambda_{j}=2 i \sin \left(\frac{2 j \pi}{2 k+1}\right), j=1,2, \ldots, 2 k, 2 k+1$. By Theorem 3.2, the assertion holds.

Remark. Note that the graph $C_{m} \times C_{n}$ can be considered as the lattice imbedded on a torus. In this case, the author of paper [6] had presented a rigorous but more complex solution to enumerate its perfect matchings.

Theorem 3.4. Let $G$ be a non-bipartite graph with a unique cycle, and $\vec{G}$ be an arbitrary orientation of $G$. Then

$$
\phi\left(P_{4} \times G\right)=\prod_{\lambda \in \lambda^{*}(\vec{G})}\left(1-3 \lambda^{2}+\lambda^{4}\right),
$$

where $\lambda^{*}(\vec{G})$ is the set of those non-negative imaginary part eigenvalues of $A(\vec{G})$.

Proof. By Theorem 2.7, $\left(P_{4} \times \vec{G}\right)^{e}$ is a Pfaffian orientation of $P_{4} \times G$. Hence, by Theorem 3.1, we have

$$
\phi\left(P_{4} \times G\right)^{2}=\operatorname{det} A\left(\left(P_{4} \times \vec{G}\right)^{e}\right) .
$$

By a suitable labeling of vertices of $\left(P_{4} \times \vec{G}\right)^{e}$, the skew adjacency matrix of $\left(P_{4} \times \vec{G}\right)^{e}$ has the following form:

$$
A\left(\left(P_{4} \times \vec{G}\right)^{e}\right)=\left(\begin{array}{cccc}
A(\vec{G}) & I & 0 & 0 \\
-I & -A(\vec{G}) & I & 0 \\
0 & -I & A(\vec{G}) & I \\
0 & 0 & -I & -A(\vec{G})
\end{array}\right)
$$

Now multiplying the first column, then the third and fourth row, then the fourth column of the partitioned matrix $A\left(\left(P_{4} \times \vec{G}\right)^{e}\right)$ by -1 , without changing the absolute value of the determinant we obtain the matrix

$$
\begin{aligned}
M & =\left(\begin{array}{cccc}
-A(\vec{G}) & I & 0 & 0 \\
I & -A(\vec{G}) & I & 0 \\
0 & I & -A(\vec{G}) & I \\
0 & 0 & I & -A(\vec{G})
\end{array}\right) \\
& =-I_{4} \otimes A(\vec{G})+B \otimes I_{n},
\end{aligned}
$$


where $\otimes$ denotes the Kronecker product of matrices and

$$
B=\left(\begin{array}{llll}
0 & 1 & 0 & 0 \\
1 & 0 & 1 & 0 \\
0 & 1 & 0 & 1 \\
0 & 0 & 1 & 0
\end{array}\right) .
$$

It is well known the eigenvalues of $-I_{4} \otimes A+B \otimes I_{n}$ are

$$
\mu_{i}-\lambda_{j} \quad(1 \leqslant i \leqslant 4, \quad 1 \leqslant j \leqslant n),
$$

where $\lambda_{1}, \lambda_{2}, \ldots, \lambda_{n}$ are the eigenvalues of $A(\vec{G})$ and $\mu_{1}, \mu_{2}, \mu_{3}$ and $\mu_{4}$ are the eigenvalues of $B$.

It is easy to calculate that the eigenvalues of $B$ are

$$
\pm \sqrt{\frac{3+\sqrt{5}}{2}}, \pm \sqrt{\frac{3-\sqrt{5}}{2}} .
$$

Thus the eigenvalues of $M$ are

$$
\pm \sqrt{\frac{3+\sqrt{5}}{2}}-\lambda_{s}, \pm \sqrt{\frac{3-\sqrt{5}}{2}}-\lambda_{s},(s=1,2, \ldots, n) .
$$

Since the determinant of the matrix $M$ is the product of these eigenvalues,

$$
\begin{aligned}
\left|\operatorname{det}\left(A\left(\left(P_{4} \times \vec{G}\right)^{e}\right)\right)\right| & =|M| \\
& =\left|\prod_{s=1}^{n}\left(\sqrt{\frac{3+\sqrt{5}}{2}}-\lambda_{s}\right)\left(-\sqrt{\frac{3+\sqrt{5}}{2}}-\lambda_{s}\right)\left(\sqrt{\frac{3-\sqrt{5}}{2}}-\lambda_{s}\right)\left(-\sqrt{\frac{3-\sqrt{5}}{2}}-\lambda_{s}\right)\right| \\
& =\prod_{s=1}^{n}\left(1-3 \lambda_{s}^{2}+\lambda_{s}^{4}\right) .
\end{aligned}
$$

If $\lambda$ is an eigenvalue of the real skew matrix $A(\vec{G})$, so is its conjugate $\bar{\lambda}$. Hence we have

$$
\begin{aligned}
\phi\left(P_{4} \times G\right) & =\sqrt{\operatorname{det}\left(A\left(\left(P_{4} \times \vec{G}\right)^{e}\right)\right)} \\
& =\prod_{s=1}^{n} \sqrt{\left(1-3 \lambda_{s}^{2}+\lambda_{s}^{4}\right)} \\
& =\prod_{\lambda \in \lambda^{*}(\vec{G})}\left(1-3 \lambda^{2}+\lambda^{4}\right),
\end{aligned}
$$

where $\lambda^{*}(\vec{G})$ is the set of those non-negative imaginary part eigenvalues of $A(\vec{G})$. The Theorem is proved. 
Similarly, by using Theorem 2.8, we can prove the following Theorem.

Theorem 3.5. Let $G$ be a non-bipartite graph with a unique cycle, and $\vec{G}$ be an arbitrary orientation of $G$. If $G$ has a perfect matching, then

$$
\phi\left(P_{3} \times G\right)=\prod_{\lambda \in \lambda^{*}(\vec{G})}\left(2-\lambda^{2}\right),
$$

where $\lambda^{*}(\vec{G})$ is the set of those non-negative imaginary part eigenvalues of $A(\vec{G})$.

Corollary 3.6. Let $G$ be a non-bipartite graph with a unique cycle, and $\vec{G}$ be an arbitrary orientation of $G$. If $G$ has a perfect matching, then $\phi\left(P_{3} \times G\right)^{2}=\phi\left(C_{4} \times G\right)$.

Proof. Corollary 3.6 is immediate from Theorem 3.2 and 3.5.

\section{Acknowledgements}

We wish to thank Professor Weigen Yan for too much useful help and advice.

\section{References}

[1] N. Biggs, Algebraic Graph Theory, Cambridge, Cambridge University Press, 1993.

[2] M. Ciucu, Enumeration of perfect matchings in graphs with reflective symmetry, J. Combin. Theory Ser. A 77(1997), 67-97.

[3] Fischer, C.H.C. Little, Even Circuits of Prescribed Clockwise Parity, Electron. J. Combin. 10 (2003) \#R45.

[4] G. G. Hall, A Graphic Model of a Class of Molecules, Int. J. Math. Edu. Sci. Technol., 4(1973), 233-240.

[5] W.Jockusch, Perfect matchings and perfect squares, J. Combin. Theory Ser. A 67(1994), $100-115$.

[6] P. W. Kasteleyn, The statistics of dimers on a lattice, Physica., 12(1961), 1209-1225.

[7] P. W. Kasteleyn, Dimer statistics and phase transition, J. Math. Phys. 4(1963), 287-293.

[8] P. W. Kasteleyn, Graph Theory and Crystal Physics. In F.Harary, editor, Graph Theory and Theoretical Physics. Academic Press, 1967, 43-110.

[9] C.H.C.Little, An extension of Kasteleyn's method of enumerating the 1-factors of planar graphs, in: D.Holton, ed., Combinatorial Mathematics, Proceedings 2nd Australian Conference, Lecture Notes in Mathematics 403 (Springer,Berlin, 1974) 63-72.

[10] L. Lovász and M. Plummer, Matching Theory, Ann. of Discrete Math. 29, North-Holland, New York, 1986.

[11] W. McCuaig, Pólya's permanent problem, Electron. J. Combin. 11 (2004), \#R79.

[12] W. McCuaig, N. Robertson, P. D. Seymour, and R. Thomas, Permanents, Pfaffian orientations, and even directed circuits (Extended abstract), Proc. 1997 Symposium on the Theory of Computing (STOC). 
[13] L. Pauling, The Nature of Chemical Bond, Cornell. Univ. Press, Ithaca, New York, 1939.

[14] J. Propp, Enumeration of Matchings: Problems and Progress, In: New Perspectives in Geometric Combinatorics (eds. L. Billera, A. Björner, C. Greene, R. Simeon, and R. P. Stanley), Cambridge University Press, Cambridge, (1999), 255-291.

[15] N. Robertson, P. D. Seymour, and R. Thomas, Permanents, Pfaffian orientations, and even directed circuits, Annals of Math., 150(1999), 929-975.

[16] R. Swinborne-Sheldrake, W. C. Herndon and I. Gutman, Kekulé structures and resonance energies of benzennoid hydrocarbons, Tetrahedron Letters, (1975), 755-758.

[17] W. Yan and F.Zhang, Enumeration of perfect matchings of graphs with reflective symmetry by Pfaffians, Adv. Appl. Math., 32(2004), 655-668.

[18] W. Yan and F. Zhang, Enumeration of perfect matchings of a type of Cartesian products of graphs, Discrete Appl. Math. 154(2006), 145-157. 